\title{
Health Literacy, Self-Care Behavior and Quality of Life in Acute Coronary Syndrome Patients: An Integrative Review
}

\author{
Kefah Zaben', Amani Khalil² \\ ${ }^{1}$ School of Nursing, Al-Quds University, Abu Dies, Palestine \\ ${ }^{2}$ School of Nursing, The University of Jordan, Amman, Jordan \\ Email: kzaben@staff.alquds.edu
}

How to cite this paper: Zaben, K. and Khalil, A. (2019) Health Literacy, Self-Care Behavior and Quality of Life in Acute Coronary Syndrome Patients: An Integrative Review. Open Journal of Nursing, 9, 383-395. https://doi.org/10.4236/ojn.2019.94035

Received: March 11, 2019

Accepted: April 14, 2019

Published: April 17, 2019

Copyright $\odot 2019$ by author(s) and Scientific Research Publishing Inc. This work is licensed under the Creative Commons Attribution International License (CC BY 4.0).

http://creativecommons.org/licenses/by/4.0/

\begin{abstract}
Purpose: The review aimed to find the best evidence on the relationship between health literacy, self-care behavior and perceived quality of life (QoL) among patients with acute coronary syndrome (ACS). Methods: Searching engines included Cumulative Index to Nursing Allied Health Literature (CINAHL), Pro Quest, MEDLINE, Google Scholar, SAGE Journals, Wiley on Line Library, and Science Direct electronic databases from 2010 to 2018. Fifteen research articles assessed the main variables and their associations with demographic and clinical variables. Results: Different methodologies and samples were analyzed: analytic descriptive, systematic review, a randomized control trial, retrospective cohort, a prospective Cohort, cross-sectional, and correlational designs. The fifteen studies showed that the patients with ACS have inadequate health literacy. Patients' ability to understand basic and complex information or to accurately assess health risks was impaired and may hinder patients' engagement in self-care. Low HL in patients having ACS is consistently associated with poor QoL. Physical domain of QoL remained the significant outcome of both self-care behavior and health literacy after adjusting for demographics and clinical variables in patients with ACS. Conclusion: The literature pointed to the importance of considering health literacy and self-care behaviors as predictors of quality of life among patients with ACS. However, there has been no previous evidence on the best process of the association between these three variables.
\end{abstract}

\section{Keywords}

Health Literacy, Self-Care Behavior, Quality of Life, Acute Coronary Syndrome 


\section{Introduction}

Cardiovascular diseases (CVDs) are a group of diseases that impact both the heart and blood vessels including coronary artery disease (CAD) and acute coronary syndrome (ACS). Acute coronary syndrome is a subcategory of CAD [1] that includes ST-segment elevation myocardial infarction (STEMI), non-ST-segment elevation myocardial infarction (NSTEMI) and unstable angina (UA) [2]. ST-segment elevation myocardial infarction is associated with the highest in-hospital mortality rate of the three conditions, while NSTEMI has the highest post-hospital discharge mortality rate. Death rates reached the highest after hospital discharge: $68 \%$ for STEMI, $86 \%$ for NSTEMI, and $97 \%$ for unstable angina [3]. Patients with ACS have the highest risk of adverse outcomes such as death, recurrent myocardial infarction (MI) readmission or heart failure during follow-up [4]. ACS almost always presents with a symptom, such as unstable angina, and is commonly linked with myocardial infarction (MI) [5]. Additionally, the incidence of ACS-related mortality is predicted to ascend as the elderly proportion of the population increases [6]. ACS costs Americans more than $\$ 150$ US billion annually, with approximately $60 \%$ - $75 \%$ of these costs related to hospital admission and readmission [7]. Evidence-based guidelines and health-system quality core measures should be considered to improve health outcome of patients with cardiovascular diseases [8].

The American Heart Association indicated that there were approximately 1.2 million hospital discharges with a diagnosis of ACS [9]. In addition, the World Health Organization (WHO) estimates that $60 \%$ of the global burden of ACS increases in developing countries [10]. ACS is a public chronic illness associated with substantial morbidity and mortality, poor QOL and frequent hospitalizations. Several factors such as treatment adherence, self-management support, poverty, health insurance, psychosocial issues were suggested as obstacles for access to proper health care in many chronic diseases [11] [12] [13] [14].

Health literacy owns skills and competencies that are needed to understand, evaluate and utilize health information and concepts for better QoL [15]. Poor health literacy hampers patients' ability to understand complex or even basic information or to accurately assess health risks, thereby impairing their ability to engage in self-care [16]. WHO reports that the term health literacy means "the cognitive and social skills which determine the motivation and ability of individuals' to gain access to, understand and use information in ways which promote and maintain good health" [17]. According to the National Assessment of Adult Literacy, health literacy means the ability to use the health-related written information to "function in society, to achieve one's goals, and to develop one's knowledge and potential". If high health literacy permits individuals to make appropriate health decisions, then it is a form of empowerment. Health literacy enables an individual to acknowledge health-related information, understand the importance of prevention and screening and be familiar with basic day to day self-care management to promote QoL [2]. Low health literacy may be an im- 
portant cause of adverse health outcomes for many chronic conditions, including ACS through the demonstrations of poor self-care behaviors [18] [19].

Acute Coronary syndrome and its various forms of presentation are associated with a reduction in the patients' own perception of QoL. While QoL is an essential measure of an effect of treatment; predictors of QoL for ACS are not completely clear and many patients suffer limitations on their physical, social and emotional performance that result in reduced health-related QoL [20]. The QoL cannot be only predicted on the foundation of the severity of the disease, management used, or the clinical status of the patient following hospital discharge [20].

Previous literature has shown an association between limited health literacy and poor knowledge about health conditions, less use of preventive services, higher hospitalization rates, increased mortality and poorer self-reported health status. The demands of understanding the importance for daily monitoring of weight and symptoms, adherence to complex regimens, physiology, and effective utilization of health care may make ACS self-care difficult in those with limited literacy. Although health literacy is associated with many adverse health outcomes, the process by which health literacy can affect the quality of life outcomes is not well understood [20] [21]. Understanding the relationship between quality of life, health literacy and self-care behaviors in patients with ACS is crucial because of the disease complexity.

Therefore, health literacy and self-care behaviors are the cornerstones for patients with ACS to live longer and attain a good QoL. However, the relationship between health literacy and ACS outcomes remains hypothesized with slight empirical evidence to support the connection. Therefore, the objective of this review is to critically appraise and review recent evidence on the associations between health literacy, self-care behaviors and QoL among patients with ACS.

\section{Materials and Methods}

The literature search was accomplished by exploring different international databases; Cumulative Index to Nursing Allied Health Literature (CINAHL), Pro Quest, MEDLINE, Google Scholar, SAGE Journals, Wiley on Line Library, and Science Direct. Search was conducted using the following keywords: self-care behaviors, acute coronary syndrome, health literacy, and QoL, risk factors and comorbidity. The search process applied keywords separately and in combination "Health literacy, self-care behavior, quality of life, and acute coronary syndrome patients" to expand, combine or exclude non-similar results.

The searching process was limited to the following inclusion criteria: 1) articles written in English, 2) published between 2010 and 2018 and, 3) different study type. Research studies and reviews were considered for the best evidence. Non-research literature and incomplete reports in the form of editorials, opinion pieces and conferences abstracts were excluded.

After evaluating and removing the duplicated articles, only 85 were found to 
be related to the topic of interest. Then, related articles were printed and read in full, following a secondary evaluation, 15 articles were exactly identified to cover the inclusion criteria. As a result, those fifteen articles were included in this review (see Figure 1). Among the fifteen research articles, an analytic descriptive $(\mathrm{n}=1)$, a retrospective cohort $(\mathrm{n}=1)$, cross-sectional $(\mathrm{n}=7)$, correlational $(\mathrm{n}=$ $2)$, systemic reviews $(\mathrm{n}=2)$, a prospective Cohort $(\mathrm{n}=1)$ and a randomized control trial $(n=1)$. The findings of each study were considered with codes for identification from the literature, summarization, synthesis and inferences, and discussion of findings for clarifying the interpretation themes.

Articles selected for retrieval were evaluated for methodological validity by two independent reviewers prior to inclusion in the review. Critical appraisal assessment and review was completed with the agreement between the reviewers and a third reviewer was consulted when required. Data were extracted from the literature using specific details about the design, populations and sample, study methods and outcomes of significance to the aim of the review. Then to show the data, all summarized pages were collected in one matrix by using word sheet.

\section{Results}

As a result, fifteen research articles were included in this review. From each article the following data have been extracted: Study purpose, design, sample and findings (Table 1) were summarized and conclusions were derived.

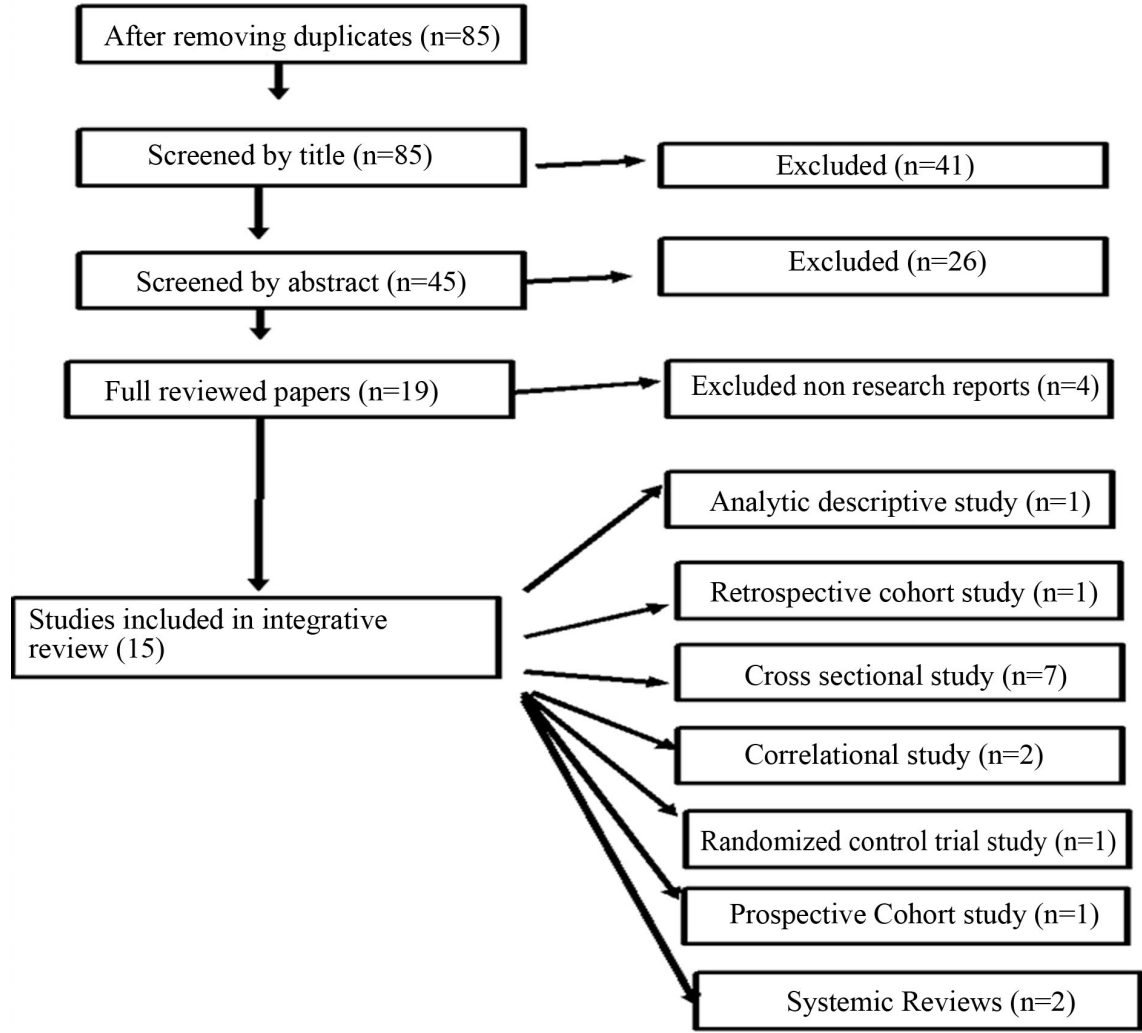

Figure 1. Search strategy and outcomes. 
Table 1. A summary of the characteristics of the included articles.

\begin{tabular}{cccc}
\hline Authors & Purpose & Design & Sample Size
\end{tabular}

De Melo Ghisi, da To explore the role of health Silva Chaves, Britto, literacy in the care of CAD \& Oh (2017) patients.

systematic review

Showing the average prevalence of low HL was $30.5 \%$. Low HL participants were more likely to be older, male, from a non-white

To evaluate the independent and interactive consequence

Martin, Schonlau, Haas, Derose, Rudd, Loucks, \& Buka, (2011). numeracy, language; oral speaking and aural listening on the calculated 10-year risk of CHD.

van Schaik, Jørstad, Twickler, Peters,

Tijssen, Essink-Bot, \& Fransen, (2017).

McNaughton, \& Kripalani, (2014).

To evaluate the association between low literacy and uncontrolled blood pressure (BP) and their associations with medication adherence.

Marzec, Carey, Lambert-Kerzner, Del Giacco, Melnyk Bryson, \& Ho, $\quad$ poor health literacy. (2015).

To investigate medication Zhong, Zheng, Guo, literacy among discharged \& Luo, (2016). patients with the acute coronary syndrome.

Prospective Cohort study $\mathrm{N}=153$

A randomized control trial

$\mathrm{N}=249$

descriptive correlational

Cross-sectiona $\mathrm{N}=423$ $\mathrm{N}=409$ language skills were highly significant association with $\mathrm{CHD}$ risk between women, and no literacy skills were associated with CHD risk in men. When evaluated together, there was some evidence for an interaction between numeracy and aural language among women suggesting that higher skills in one area (e.g., aural language) may compensate for difficulties in another resulting in an equally low risk of $\mathrm{CHD}$.

Inadequate health literacy is predominant in CAD patients in the Netherlands, and is associated with less favorable CVD risk profiles. Where many different forms of CVD prevention were unsuccessful, and nurse coordinated care appear to be influential among patients with inadequate health literacy.

About 192 (45\%) patients had low literacy and 227 (52.9\%) had uncontrolled blood pressure. after adjusting for the following factors; age, gender, race, employment, education, mental status, and self-reported adherence, low literacy was associated with uncontrolled BP (OR 1.75, 95\% CI 1.06 - 2.87). Lower self-reported adherence was associated with uncontrolled BP; the relationship between refill adherence and uncontrolled $\mathrm{BP}$ was not statistically significant.

Cognitive dysfunction and risk for poor health literacy are common in patients hospitalized with ACS. The study suggests an association between mild neurocognitive disorder and medication non-adherence in the usual care group but not in the intervention group.

Showed that patients with Acute Coronary Syndrome who were investigated for medication literacy was insufficient; $>20 \%$ did not have sufficient knowledge on the types of drugs and the frequency that they need to take the drugs, $>30 \%$ did not know the name of and the dosage of the drugs they are taking, and $>70 \%$ did not have adequate knowledge of the effects and side effects of the drugs they are taking.

Medication adherence rates at one-year in patients after hospital

Bailey, Fang, Annis, literacy, and to examine the O'Conor, relationship between derived Retro-spective Paasche-Orlow, health literacy estimates and cohort study Wolf, (2015). discharge for an ACS ranged about $54 \%$ and $86 \%$, no consistent predictors of non-adherence through all cardiac medication categories, and the medication adherence to secondary prevention pharmacotherapy was less than the highest standard in patients after hospital discharge for an ACS.

To assess the validity of a predictive model of health

\section{secondary prevention}

pharmacotherapy in adults after hospital discharge.

A systematic review

$\mathrm{N}=$ five electronic databases

After adjusting for demographic and clinical characteristics, the risk of readmission was $12 \%$ lower and the incidence rate $16 \%$ lower for patients with above basic literacy, And was found to be a significant, independent predictor of readmissions. As a modifiable risk factor with evidence-based solutions, health literacy should be considered in readmission reduction efforts. 


\section{Continued}

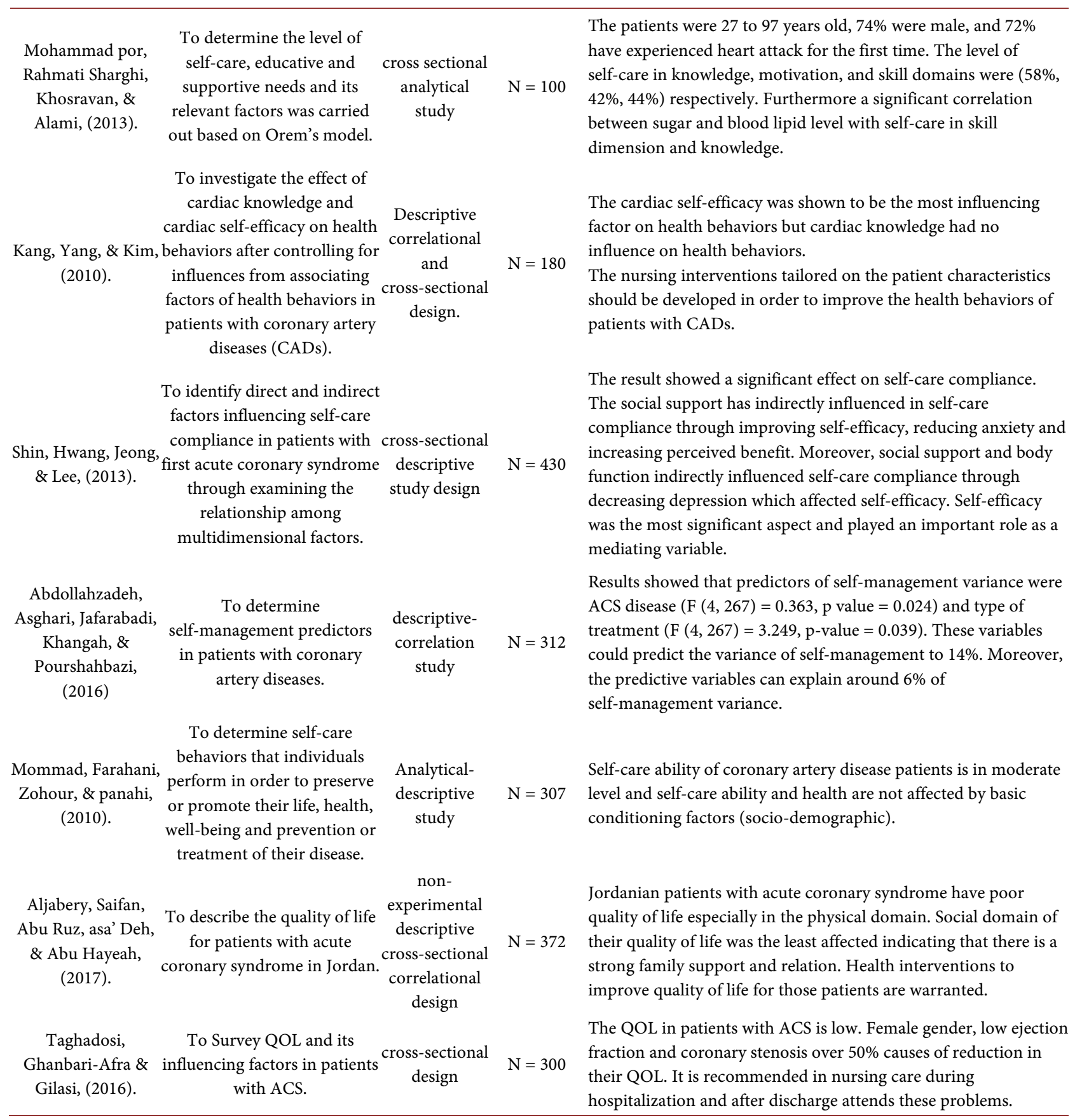

\subsection{Health Literacy}

Studies explored the epidemiology, risk factors and outcomes that are connected to it in patients with CAD. The average prevalence of health literacy was $30.5 \%$, which was considered as low. Participants with low health literacy were more likely to be older, male, non-white, have many CVD comorbidities, lower educational level, disadvantaged socioeconomic position and unemployed. Low health literacy was consistently associated with hospital readmissions, low health related QoL, higher anxiety and lower social support. Many health behaviors and 
outcomes were involved such as diet, exercise, smoking, medication use, hospital readmission, knowledge and psychosocial indicators. Finally, it could be speculated that health literacy is very limited in CAD patients and healthcare providers. Health policies should adopt strategies that can potentially improve and mitigate the impact of low health literacy in the care of CAD patients [22].

For adequate level of health literacy, patient with $C D$ should have skills on reading, numeracy, language; oral speaking and aural listening. A descriptive correlation study evaluated the independent and interactive consequence of these four literacy skills on the calculated 10-year risk of CHD. Health literacy in reading, numeracy, and aural language skills were highly significantly associated with CHD risk in women compared to men. An interaction between numeracy and aural language among women suggested that higher skills in one area (e.g., aural language) may compensate for difficulties in another resulting in an equally low risk of CHD. The evidence indicates that there is an independent and interactive effect of literacy skills on decreasing the risk of CHD and CVD in different nationality [23]. Authors highlight the need for the development of easy-to-use assessments of the oral exchange in the healthcare setting and the need to better understand which literacy skills are most important for a given population and health outcome [22].

Health literacy was assessed using different objective and subjective instruments. The short Rapid Estimate of Adult Literacy in Medicine (REALM-D) instrument showed inadequate health literacy in patients with CAD in Netherlands, and was associated with less favorable CVD risk profiles [24]. Low health literacy was associated with uncontrolled blood pressure (BP) and poor medication adherence among urban participants with hypertension and coronary disease $(\mathrm{N}=423)$ [25]. Nurses need to coordinate communicative care as a preventive management through improving patients understanding and ability to raise self-care and adherence.

Health literacy is reflected through neurocognitive dysfunction in patients hospitalized with ACS [26]. A Prospective Cohort study was conducted in China to investigate medication literacy and to determine the importance of medication literacy among discharged patients with ACS. The demographic and clinical data of patients were obtained from hospital record. Medication literacy was assessed by interviewing patients in the 7 to 30 days after discharge from the hospital. Medication literacy was found to be insufficient in $>20 \%$ on types and the frequency of drugs, $>30 \%$ on name of and the dosage of the drugs and $>70 \%$ on the effects and side effects of the drugs they are taking. The study indicated that medication literacy scores decreased with age but increased with education [27].

A systematic review was conducted on patients with ACS. Synthesized current evidence on medication adherence rates and related risk factors was performed to assess adherence to pharmacotherapy in adults after hospital discharge. The results showed medication adherence rates ranged between $54 \%$ and $86 \%$ at one-year in patients after hospital discharge for an ACS. No consistent predictors of non-adherence were found through all cardiac medication categories 
[28]. A retrospective design study was conducted to evaluate the validity of a predictive model of health literacy, and to test the relationship between derived health literacy estimates and 30-day hospital readmissions for acute myocardial infarction (AMI). About 696 participants out of 7733 Medicare hospitalized patients with AMI responded to three health literacy tools (Functional Health Literacy in Adults, Newest Vital Sign, and Rapid Estimate of Adult Literacy in Medicine). After controlling for demographic and clinical characteristics, the risk of 30 -day readmission was $12 \%$ lower $(\mathrm{p}=0.03)$ and the incidence rate $16 \%$ lower $(\mathrm{p}<0.01)$ for patients with a literacy score above basic. Inadequate health Literacy was found to be a significant, independent predictor of 30-day readmissions. As a modifiable risk factor with evidence-based solutions, health literacy should be considered in readmission reduction efforts [29].

\subsection{Self-Care}

Patients with MI have deficit in their knowledge, skills and motivation toward better self-care and health-related outcomes. Knowledge, motivation and skills expressed in self-care questionnaire were scored as follow: $58 \%, 44 \% 42 \%$, respectively. Orem theory was used to assess the contributions of these variables among patients with MI. They found that patients suffering from low self-care, knowledge and motivation experienced heart attack for the first time [30]. Others examined the effect of knowledge and self-efficacy on health behaviors after controlling of factors of health behaviors among Korean patients with CAD. They showed low cardiac knowledge, moderately high cardiac self-efficacy and moderate level of health behaviors. Although, that these interrelated variables were assessed but the nature of the relationship between knowledge, skills and motivation to self-care never been illustrated enough. It was suggested that nursing interventions tailored to the patient characteristics should be developed in order to improve the health behaviors of patients with CADs [31].

Adherence to treatment regimen is a multidimensional process in patients with ACS. Self-care, compliance and adherence are all used interchangeably in the literature. Concepts such as social support and body function through enhancing self-efficacy, reducing anxiety, increasing perceived benefit and reducing depression correlated significantly in patients with ACS. Self-efficacy was played a mediating role between self-care adherence and depression and social support. Additional, anxiety and perceived benefits mediated the relationship between self-care compliance and social support [32]. Other explored self-management predictors in Tabriz, Iran. Standard self-management questionnaire (Patient Activation Measure) showed approximately half of patients have good self-management. Moreover, they found that self-management activities were different based on the history of disease and type of treatment applied. Furthermore, self-management behaviors were more favorable among patients with ACS compared to patients with high cholesterol and blood pressure. It was proposed that more efforts are needed to encourage self-management of patients with ACS [33]. Patients with 
CAD had moderate level in their self-care ability. Although, socio-demographic showed non-significant association with self-care behaviors, self-care behavior was considered important predictor of health related outcomes. A Solid conceptual framework or model of self-care behavior and other important related variables can help in improving care [34].

\subsection{Quality of Life}

Jordanian patients with ACS were found to have poor quality of life domains. The three domains of QoL are physical, emotional and social. The MacNew heart disease health-related Quality of life (MacNew) showed that physical domain was the mostly impaired and social domain scored the least. Quality of life varied based on gender, level of education, employment status, physical activity, history of dyslipidemia and medical diagnosis. They found that patients suffered from poor quality of life in the three domains among patients with ACS. Health interventions are necessary to improve quality of life for those patients [35].

Others assessed QOL and its associates among patients with ACS in Iran. Quality of life scored below the mean in $58.3 \%$ of patients. The mental component of QOL scored higher than physical component. The QOL score in physical component was $51.57 \pm 24.56$ (physical limits, physical performance, pain, public health) and the psychological component was $61.15 \pm 19.46$ (energy, social performance, emotional limitation, mental health). In addition they found Low QoL correlated significantly with aging. Gender female (42.3\%) and male (57.7\%) male gender were associated with a 1.88 times increase in QoL. Quality of life was lower after 2 months of experiencing ACS. Coronary stenosis increased 3.25 times as likely to the desirable QoL. Furthermore, normal ejection fraction increased 3.4 times as likely to desirable QoL. Female gender, low ejection fraction and coronary stenosis explained 50\% decreased in QoL in patients with ACS. They proposed that nursing care during hospitalization and after discharge needs to address the methods to improve the QoL [36].

\section{Discussion}

In this integrative review, variables of health literacy, self-care behavior, and quality of life were explored through a review of studies revealed patients with acute coronary syndrome. Modifiable risk factors and health literacy should be considered in readmission reduction efforts. Health behaviors and outcomes were studied include diet, exercise, smoking, medication use, hospital readmission and knowledge. Quality of life has different domains; physical, mental, environmental and psychosocial.

Patients with ACS showed low average prevalence of HL (30.5\%). Patients with low HL had lower educational levels, disadvantaged socioeconomic and were less likely to be employed. Low HL was consistently associated with hospital readmissions, low health-related to poor quality of life, higher anxiety and lower social support [22] [29]. Inadequate health literacy is predominant in CAD 
patients; Low literacy is associated with uncontrolled BP awareness [24] [25]. Cognitive dysfunction and risk for poor health literacy are common in patients hospitalized with ACS [26]. Medication literacy scores decreased with age and increased with education among participants with ACS after discharged [27]. Literacy was found to be a significant, independent predictor of readmissions for acute myocardial infarction (AMI) and was $12 \%$ lower $(\mathrm{p}=0.03)$ and the incidence rate $16 \%$ was lower $(\mathrm{p}<0.01)$ for patients with a literacy score of above basic [29].

Several studies explored the relationship of the level of self-care, educative and supportive needs and other relevant factors, was assessed using questionnaires based on Orem's model in various domains such as knowledge, motivation, and skill. The highest level of self-care was $58 \%$ scored in the knowledge domain, and then was $44 \%$ scored in skill domain, and the lowest was $42 \%$ scored in the motivation domain [30]. Other studies explore the importance of cardiac knowledge and health behaviors in patients with CADs. The outcome presented relatively low cardiac-related knowledge, and a moderate level of health behaviors [31]. Other studies showed that social support indirectly affected self-care compliance, self-care ability of coronary artery disease patients in the moderate level and no affects socio-demographic on self-care ability with coronary artery disease [32] [33] [34].

Furthermore, one study showed that the physical domain of the quality of life was the most affected followed by the emotional and lastly was the social domain [35]. The QOL in patients with ACS low [36]. The literature showed important relationships between the variables; self-care behavior, health literacy and quality of life among patients with acute coronary syndrome, however, these studies mostly described the variables as the following; health literacy and quality of life, self-care and quality of life, and, health literacy and self-care separately. Until now, no one has studied the three variables together. By searching the interrelated effects of self-care behavior on the relationship between health literacy and quality of life, the results indicated of a potential mediating, moderating or other potential mechanism of action between these variables. More research studies are needed to test the nature of the relationship of those crucial variables to improve the patients' outcomes.

\section{Conclusion}

The literature showed health literacy, self-care behaviours and quality of life separately among ACS patients. The findings indicated that a gap of knowledge is present on the mediating effect of self-care behaviours on the relationship of health literacy and perceived quality of life among patients with ACS. Few studies discussed specifically patient with ACS while majority discussed these variables in patients with CAD.

\section{Conflicts of Interest}

The authors declare no conflicts of interest regarding the publication of this paper. 


\section{References}

[1] World Health Organization (2011) Global Atlas on Cardiovascular Disease Prevention and Control. Geneva.

[2] Katus, H., Ziegler, A., Ekinci, O., Giannitsis, E., Stough, W.G., Achenbach, S., Crea, F., et al. (2017) Early Diagnosis of Acute Coronary Syndrome. European Heart Journal, 38, 3049-3055. https://doi.org/10.1093/eurheartj/ehx492

[3] Anderson, J.L. and Morrow, D.A. (2017) Acute Myocardial Infarction. New England Journal of Medicine, 376, 2053-2064. https://doi.org/10.1056/NEJMra1606915

[4] Gershlick, A.H., Khan, J.N., Kelly, D.J., Greenwood, J.P., Sasikaran, T., Curzen, N., Wang, D., et al. (2015) Randomized Trial of Complete versus Lesion-Only Revascularization in Patients Undergoing Primary Percutaneous Coronary Intervention for STEMI and Multivessel Disease: The CvLPRIT Trial. Journal of the American College of Cardiology, 65, 963-972. https://doi.org/10.1016/j.jacc.2014.12.038

[5] Lippi, G., Cervellin, G. and Sanchis-Gomar, F. (2016) Energy Drinks and Myocardial Ischemia: A Review of Case Reports. Cardiovascular Toxicology, 16, 207-212. https://doi.org/10.1007/s12012-015-9339-6

[6] Chen, A.M., Yehle, K.S., Albert, N.M., Ferraro, K.F., Mason, H.L., Murawski, M.M. and Plake, K.S. (2013) Health Literacy Influences Heart Failure Knowledge Attainment But Not Self-Efficacy for Self-Care or Adherence to Self-Care over Time. Nursing Research and Practice, 2013, Article ID: 353290. https://doi.org/10.1155/2013/353290

[7] Zhao, Z. and Winget, M. (2011) Economic Burden of Illness of Acute Coronary Syndromes: Medical and Productivity Costs. BMC Health Services Research, 11, 35. https://doi.org/10.1186/1472-6963-11-35

[8] Page, R.L., II, V.G., Van Den Bos, J., Gray, T.J., Hoetzer, G.L., Bhandary, D. and Nair, K.V. (2016) The Cost of Inpatient Death Associated with Acute Coronary.

[9] Roger, V.L., Go, A.S., Lloyd-Jones, D.M., Benjamin, E.J., Berry, J.D., Borden, W.B., Fullerton, H.J., et al. (2012) Heart Disease and Stroke Statistics-2012 Update: A Report from the American Heart Association. Circulation, 125, e2-e220.

[10] Sanchis-Gomar, F., Perez-Quilis, C., Leischik, R. and Lucia, A. (2016) Epidemiology of Coronary Heart Disease and Acute Coronary Syndrome. Annals of Translational Medicine, 4, 256. https://doi.org/10.21037/atm.2016.06.33

[11] Custer, A.M., Walter, M. and Scheier, L.M. (2016) Web-Based Interventions for Treating Youth with Asthma: Is Efficacy on the Horizon.

[12] Scarborough, P., Wickramasinghe, K., Bhatnagar, P. and Rayner, M. (2011) Trends in Coronary Heart Disease 1961-2011. British Heart Foundation, London.

[13] Khalil, A., Darawad, M., Algamal, E. and Mansour, A. (2012) Predictors of Dietary and Fluid Nonadherence in Jordanian Patients with End-Stage Renal Disease Receiving Hemodialysis: A Cross-Sectional Study. Journal of Clinical Nursing, 22, 127-136. https://doi.org/10.1111/j.1365-2702.2012.04117.x

[14] Khalil, A. and Abdalrahim, M. (2014) Knowledge, Attitudes, and Practices towards Prevention and Early Detection of Chronic Kidney Disease. International Nursing Review, 61, 237-245. https://doi.org/10.1111/inr.12085

[15] Wilson, F.L., Mood, D. and Nordstrom, C.K. (2010) The Influence of Easy-to-Read Pamphlets about Self-Care Management of Radiation Side Effects on Patients' Knowledge. Oncology Nursing Forum, 37, 774-781. https://doi.org/10.1188/10.ONF.774-781

[16] Nielsen, K.K., Damm, P., Bygbjerg, I.C. and Kapur, A. (2017) Barriers and Facilita- 
tors for Implementing Programmes and Services to Address Hyperglycaemia in Pregnancy in Low and Middle Income Countries: A Systematic Review. Diabetes Research and Clinical Practice, 145, 102-118.

[17] The World Health Organization WHO (2013).

[18] Fabbri, M., Yost, K., Rutten, L.J.F., Manemann, S.M., Boyd, C.M., Jensen, D., Roger, V.L., et al. (2018) Health Literacy and Outcomes in Patients with Heart Failure: A Prospective Community Study. Mayo Clinic Proceedings, 93, 9-15. https://doi.org/10.1016/j.mayocp.2017.09.018

[19] Kato, N., Jaarsma, T. and Gal, T.B. (2014) Learning Self-Care after Left Ventricular Assist Device Implantation. Current Heart Failure Reports, 11, 290-298. https://doi.org/10.1007/s11897-014-0201-0

[20] Bakas, T., McLennon, S.M., Carpenter, J.S., Buelow, J.M., Otte, J.L., Hanna, K.M., Welch, J.L., et al. (2012) Systematic Review of Health-Related Quality of Life Models. Health and Quality of Life Outcomes, 10, 134. https://doi.org/10.1186/1477-7525-10-134

[21] Berkman, N.D., Sheridan, S.L., Donahue, K.E., Halpern, D.J. and Crotty, K. (2011) Low Health Literacy and Health Outcomes: An Updated Systematic Review. Annals of Internal Medicine, 155, 97-107. https://doi.org/10.7326/0003-4819-155-2-201107190-00005

[22] Song, I.G., Lee, H., Yi, J., Kim, M.S., Kawachi, I. and Park, S.M. (2018) Health Literacy and Use of Preventive Health Services among North Korean Defectors in the Republic of Korea. PLOS ONE, 13, e0195964. https://doi.org/10.1371/journal.pone.0195964

[23] De Melo Ghisi, G.L., da Silva Chaves, G.S., Britto, R.R. and Oh, P. (2017) Health Literacy and Coronary Artery Disease: A Systematic Review. Patient Education and Counseling, 101, 177-184.

[24] Martin, L.T., Schonlau, M., Haas, A., Derose, K.P., Rudd, R., Loucks, E.B., Buka, S.L., et al. (2011) Literacy Skills and Calculated 10-Year Risk of Coronary Heart Disease. Journal of General Internal Medicine, 26, 45-50. https://doi.org/10.1007/s11606-010-1488-5

[25] McNaughton, C.D., Jacobson, T.A. and Kripalani, S. (2014) Low Literacy Is Associated with Uncontrolled Blood Pressure in Primary Care Patients with Hypertension and Heart Disease. Patient Education and Counseling, 96, 165-170. https://doi.org/10.1016/j.pec.2014.05.007

[26] Marzec, L.N., Carey, E.P., Lambert-Kerzner, A.C., Del Giacco, E.J., Melnyk, S.D., Bryson, C.L., Ho, P.M., et al. (2015) Cognitive Dysfunction and Poor Health Literacy Are Common in Veterans Presenting with Acute Coronary Syndrome: Insights from the Medication Study. Patient Preference and Adherence, 9, 745.

https://doi.org/10.2147/PPA.S75110

[27] Zhong, Z., Zheng, F., Guo, Y. and Luo, A. (2016) Medication Literacy in a Cohort of Chinese Patients Discharged with Acute Coronary Syndrome. International Journal of Environmental Research and Public Health, 13, 720. https://doi.org/10.3390/ijerph13070720

[28] Chen, H.Y., Saczynski, J.S., Lapane, K.L., Kiefe, C.I. and Goldberg, R.J. (2015) Adherence to Evidence-Based Secondary Prevention Pharmacotherapy in Patients after an Acute Coronary Syndrome: A Systematic Review. Heart \& Lung. The Journal of Acute and Critical Care, 44, 299-308. https://doi.org/10.1016/j.hrtlng.2015.02.004

[29] Hirschman, K., Shaid, E., McCauley, K., Pauly, M. and Naylor, M. (2015) Continuity of Care: The Transitional Care Model. OJIN: The Online Journal of Issues in 
Nursing, 20, 1.

[30] Mohammad por, A., Rahmati Sharghi, N., Khosravan, S. and Alami, A. (2013) Investigating the Level of Self-Care and Supportive Educative Needs of Patients with Myocardial Infarction, Based on Orem's Model. Journal of Research and Health, 3 , 489-496.

[31] Kang, Y., Yang, I.S. and Kim, N. (2010) Correlates of Health Behaviors in Patients with Coronary Artery Disease. Asian Nursing Research, 4, 45-55. https://doi.org/10.1016/S1976-1317(10)60005-9

[32] Shin, E.S., Hwang, S.Y., Jeong, M.H. and Lee, E.S. (2013) Relationships of Factors Affecting Self-Care Compliance in Acute Coronary Syndrome Patients Following Percutaneous Coronary Intervention. Asian Nursing Research, 7, 205-211. https://doi.org/10.1016/j.anr.2013.10.003

[33] Abdollahzadeh, F., Asghari, E., Jafarabadi, M.A., Khangah, B. and Pourshahbazi, E. (2016) Predictors of Self-Management in Patients with Coronary Artery Diseases. International Journal of Medical Research \& Health Sciences, 5, 637-643.

[34] Mohammad, H.M., Farahani, B., Zohour, A.R. and Panahi, A.S. (2010) Self-Care Ability Based on Orem's Theory in Individuals with Coronary Artery Disease.

[35] Hayeah, H.M.A., Saifan, A.R., AbuRuz, M.E. and Aljabery, M.A. (2017) Health-Related Quality of Life in Heart Failure in Jordan from Patient's Perspectives.

[36] Afra, L.G., Taghadosi, M. and Gilasi, H.R. (2016) Relationship between Ischemic Heart Disease and Sexual Satisfaction. Global Journal of Health Science, 8, 263. https://doi.org/10.5539/gjhs.v8n1p263 